\title{
EFFECT OF SOME ANTIOXIDANTS ON GROWTH, YIELD AND SOME CHEMICAL CONSTITUENTS OF ONION (ALLIUM CEPA L.) PLANTS
}

\author{
Mohamed, A. Seif El-Yazal
}

Dep. of Agric. Botany, Fac. of Agric. Fayoum, Univ., Egypt.

\section{ABSTRACT}

The present investigation was conducted during the two successive seasons 2004/2005 and 2005/2006 on onion (Allium cepa L. $\mathrm{cv}$. Giza 20) grown at the experimental station of the Faculty of Agriculture, Fayoum University, Egypt, to study the effect of some antioxidants (ascorbic acid, citric acid, oxalic acid and salicylic acid) foliar spray at the rate of $0,2.5,5$ and $10 \mathrm{mM}$ on the possibility of improving growth, yield and some chemical constituents of onion plants. Significant positive influences of all antioxidants treatments used in this study were observed on growth, yield of bulb and some chemical composition characters in both tested seasons. The obtained results indicated that, the growth characters, i.e. plant height, number of leaves/plant, and fresh and dry weight of leaves/plant were affected positively by the application of all antioxidants used at all studied rates. The maximum increase in this respect was obtained by the treatment of $10 \mathrm{mM}$ followed by $5 \mathrm{mM}$ treatment as compared to the control untreated plants in both seasons. The same treatments increased bulb yield and its components (bulb diameter, as well as fresh and dry weight of bulb). In addition the most effective treatments for enhancing the concentration of chlorophyll a and $b$, total caroteniods, total free amino acid, total indoles, and total soluble phenols on fresh leaves, and total carbohydrates, total and reducing sugars, anthocyanin concentration, crude protein and some nutrients i.e. N,P and $\mathrm{K}$ in dry matter of leaves and the content of bulbs from total soluble solids (T.S.S.\%), total soluble carbohydrates and protein was observed at higher concentration $(10 \mathrm{mM})$ of all antioxidants used especially Ascorbic acid and Salicylic acid. Thus, the highest application of antioxidants is recommended for improving growth, yield and chemical composition of onion plants.

Key words: Onion (Allium cepa L.), antioxidants (Ascorbic acid, Citric acid, Oxalic acid and Salicylic acid), growth, yield, chemical constituents

\section{INTRODUCTION}

Onion (Allium cepa L.) is one of the most important vegetable crops in Egypt, since it is one of the sources for hard currency, due to the early availability of the crop for foreign market as well as its higher quality compared with other onions. Nowadays, there is a widespread use of natural and safety substances such as antioxidants particularly, ascorbic acid, citric acid, oxalic acid and salicylic acid for enhancing or improving growth and productivity of many crops for the aim of reducing health hazards. Since, antioxidants has synergistic effect on growth, yield and chemical composition under favorable and unfavorable environmental conditions. These compounds as non enzymatic material have beneficial effect on catching the free radical or the active oxygen

Fayoum J. Agric. Res. \& Dev., Vol. 21, No. 1, January, 2007 
species namely singlet oxygen, superoxide anion, hydrogen peroxide, hydroxyl radicals and ozone that producing during photosynthesis and respiration process (Zhang and Klessing, 1997). Leaving these free radicals without chelating or catching leads to lipids oxidation and the loss of plasma membrane permeability and the death of cell within plant tissues. They also have an auxinic action (Prusky, 1988; Elade, 1992; Raskin 1992 and Buehala and Schmid, 1997). The beneficial effect of antioxidants on growth and yield of several plant species was reported by several workers (Zaghlool et al., 2001 on dry bean; Ismail and Fawzy, 2001 on pea seedling; Ali 2001 and 2002 on tomato plant; Gad EL-Hak et al., 2003 on potato, Abd El-Magid et al., 2004 and Shalaby, 2006 on onion; Zaghlool et al., 2006 on wheat and Rady, 2006 on sunflower plants).

Accordingly, the present work was planned to study the effect of exogenous application of ascorbic acid, citric acid, oxalic acid or salicylic acid at different rates on growth and yield, as well as some chemical constituents of onion plants during different stages of growth at both 2004/2005 and 2005 /2006 seasons.

\section{MATERIALS AND METHODS}

A field experiment was conducted using onion plants (cv. Giza 20) during two successive seasons 2004/2005 and 2005/2006 at experimental area of Faculty of Agriculture, Fayoum University, Egypt. Before planting the most important physical and chemical characters of the selected soil was determined (Table 1) according to Wilde et al., (1985).

During the experiment, different treatments were arranged in randomized complete design with three replications. The experimental plot was $4 \times 6 \mathrm{~m}$ contained 10 rows each was $4 \mathrm{~m}$ long and $50 \mathrm{~cm}$ width. The outside rows of each plot was considered as boarders. The central two rows of each plot were assigned for plant sampling. Thirty five days old onion transplants were planted $10 \mathrm{~cm}$ apart in the two sides of each row on $25^{\text {th }}$ November in both seasons. The plants were sprayed with tap water (as a control) or ascorbic acid or citric acid or oxalic acid or salicylic acid at the rates of $(2.5,5$ and 10 $\mathrm{mM}$ ) respectively. Few drops of Tween-20 were added to the spraying solution as a surfactant. The foliar applications were carried out at 40 and 55 days after transplanting. Thus, the experiment involved thirteen treatments as follows:

1- Control

2- Ascorbic acid at $2.5 \mathrm{mM}$

3- Ascorbic acid at $5 \mathrm{mM}$

4- Ascorbic acid at $10 \mathrm{mM}$

5-Citric acid at $2.5 \mathrm{mM}$

6- Citric acid at $5 \mathrm{mM}$

7- Citric acid at $10 \mathrm{mM}$
8-Oxalic acid at $2.5 \mathrm{mM}$

9- Oxalic acid at $5 \mathrm{mM}$

10- Oxalic acid at $10 \mathrm{mM}$

11- Salicylic acid at $2.5 \mathrm{mM}$

12- Salicylic acid at $5 \mathrm{mM}$

13- Salicylic acid at $10 \mathrm{mM}$

All antioxidants were applied as foliar application. 
EFFECT OF SOME ANTIOXIDANTS ON GROWTH, YIELD.

Table (1): The physical and chemical analysis of used soil before planting in both seasons.

\begin{tabular}{|c|c|c|c|c|c|}
\hline Properties & $2004 / 2005$ & $2005 / 2006$ & Properties & $2004 / 2005$ & $2005 / 2006$ \\
\hline \multicolumn{7}{|c|}{ Physical roperties } \\
\hline Sand \% & 26.80 & 27.00 & Silt \% & 25.80 & 26.20 \\
\hline Clay \% & 46.40 & 46.80 & Texture grade & Clay & clay \\
\hline \multicolumn{7}{|c|}{ Chemical properties } \\
\hline Organic matter \% & 1.76 & 1.75 & $\mathrm{pH}(1: 2.5)$ & 7.33 & 7.26 \\
\hline Ec(dsm) & 0.61 & 0.64 & CaCO $\%$ & 5.60 & 5.49 \\
\hline \multicolumn{7}{|c|}{ Available macro-and micro-elements (ppm) } \\
\hline $\mathrm{N}$ & 473.00 & 479.00 & $\mathrm{P}$ & 418.00 & 425.00 \\
\hline $\mathrm{K}$ & 21.00 & 24.00 & $\mathrm{Fe}$ & 85.00 & 83.00 \\
\hline $\mathrm{Zn}$ & 27.90 & 27.00 & $\mathrm{Mn}$ & 3.40 & 3.10 \\
\hline $\mathrm{Cu}$ & 0.79 & 0.75 & & & \\
\hline
\end{tabular}

All onion plants including control were fertilized with NPK using the dose recommended by Ministry of Agriculture, Egypt. Phosphorous as triple calcium super phosphate $\left(46 \% \mathrm{P}_{2} \mathrm{O}_{5}\right)$ at the rat of $75 \mathrm{~kg} / \mathrm{fed}$., was mixed with soil before sowing. Nitrogen fertilizer was applied in the form of urea $(46 \% \mathrm{~N})$ at the rate of $250 \mathrm{~kg} / \mathrm{fed}$., and $50 \mathrm{~kg} / \mathrm{fed}$., of potassium sulphate $\left(48 \% \mathrm{~K}_{2} \mathrm{O}\right)$. The amounts of $\mathrm{N}$ and $\mathrm{K}$ fertilizers were divided into two equal doses, the first was added after 30 days from transplanting and the second was applied one month later. After 90 days from transplanting a random sample of mature fully expanded leaves of 10 plants was taken from each plot to measure, i.e. plant height $(\mathrm{cm})$, number of leaves/plant, as well as fresh and dry weight of leaves/plant. Onion plants were grown to maturity and they were considered mature when the foliage lodged on $50 \%$ of the plants (El-Sayed and Attia, 1999). At this stage of maturity (185 days) from transplanting, diameter of bulb $(\mathrm{cm})$, weight of bulb $(\mathrm{g})$, fresh and dry weight of bulb $(\mathrm{g})$ and total bulbs yield (ton/fed.,) were estimated for the remained 2 rows. Samples of bulb were taken at random and dried, then kept for chemical analysis.

\section{Chemical analysis}

Fresh and dried leaves and bulbs (90 days of old plants for leaves and maturity stage for bulbs) were used for the determination of the following constituents: In fresh bulbs only total soluble solids (T.S.S.) was estimated using handle refractometer model PZONr. 19877 (A.O.A.C. 1995). Photosynthetic pigments: chlorophyll $\mathrm{a}, \mathrm{b}$ and caroteniods were extracted from fresh leaves by acetone $(80 \%)$ then, their concentrations were determined $\mu \mathrm{g} / \mathrm{g}$ fresh weight according to Welburn and Lichtenthaler (1984), Total carbohydrates mg/g dry weight were determined colorimetrically according to the method described by Herbert et al., (1971). Total and reducing sugars were determined according to A.O.A.C. (1995), and recorded as $\mathrm{mg} / \mathrm{g}$ dry weight, Anthocyanin concentration $\mathrm{mg} / 100 \mathrm{~g}$ dry weight was determined according to the method described by Hoagland (1980). Total free amino acids in fresh leaves were determined colorimetrically according to the method described by Jayarman (1981) and recorded as $\mathrm{mg} / \mathrm{g}$ dry weight. Total indoles $\mathrm{mg} / \mathrm{g}$ fresh weight were determined according to Larson $\boldsymbol{e t}$ al. (1962), Total soluble phenols in fresh leaves were determined according to A.O.A. C. (1995) and recorded as $\mathrm{mg} / \mathrm{g}$ dry weight. Nitrogen $\%$, and crude protein percentage was determined according to micro Kjeldahl as described by A.O.A.C. (1995), phosphorus \% was determined

Fayoum J. Agric. Res. \& Dev., Vol. 21, No. 1, January, 2007 
according to A.O.A.C. (1995), potassium was determined by Flame Photometer, Parkin-Elmer model 52 according to Page et al. (1982).

\section{Statistical analysis:}

The experiment was in a complete randomized block design with 13 treatment and 4 replicates for each treatment. Results were statistically analyzed using the L.S.D. at probability level of 5\% for comparisons (Gomez and Gomez 1983).

\section{RESULTS:}

A - Growth characters:

Data presented in Table (2) indicate that, growth parameters of onion plants represented as plant height, number of leaves/plant, as well as fresh and dry weight of leaves/plant were greatly improved by antioxidants application at different rates. The highest increase in growth parameters were observed when onion plants were sprayed with the highest rate of antioxidant $(10 \mathrm{mM})$ of either ascorbic acid or salicylic acid. Such increases reached 13.41and 13.40\% respectively on plant height in the first season and 15.62 and $15.18 \%$ respectively in the second season in comparison to the control untreated plants. Similar trend was observed on the number of leaves/plant in which the increase reach to 71.24 and $70.91 \%$ respectively in the first season and 55.62 and $53.55 \%$ respectively in the second season. Also, fresh weight of leaves/plant increased by 16.35 and $15.35 \%$ respectively in the first season and 17.98 and $17.86 \%$ respectively in the second season. Moreover, dry weight of leaves/ plant were increased by 36.81 and $36.26 \%$ respectively in the first season and 38.20 and $37.19 \%$ respectively in the second season as compared to the control untreated plants.

Table (2): Effect of some antioxidants on growth characters of onion plants during 2004/2005 and 2005/2006 seasons.

\begin{tabular}{|c|c|c|c|c|c|c|c|c|c|}
\hline \multirow[t]{2}{*}{ Treat. } & \multirow[t]{2}{*}{$\begin{array}{l}\text { Conc } \\
(\mathrm{mM})\end{array}$} & \multicolumn{2}{|c|}{$\begin{array}{l}\text { Plant height } \\
(\mathrm{cm})\end{array}$} & \multicolumn{2}{|c|}{$\begin{array}{c}\text { Number of leaves/ } \\
\text { plant }\end{array}$} & \multicolumn{2}{|c|}{$\begin{array}{c}\text { Fresh weight of } \\
\text { leaves/plant }\end{array}$} & \multicolumn{2}{|c|}{$\begin{array}{c}\text { Dry weight of } \\
\text { leaves/plant }(\mathrm{g})\end{array}$} \\
\hline & & $\begin{array}{c}\text { First } \\
\text { season }\end{array}$ & $\begin{array}{l}\text { Second } \\
\text { season }\end{array}$ & $\begin{array}{c}\text { First } \\
\text { season }\end{array}$ & $\begin{array}{l}\text { Second } \\
\text { season }\end{array}$ & $\begin{array}{c}\text { First } \\
\text { season }\end{array}$ & $\begin{array}{l}\text { Second } \\
\text { season }\end{array}$ & $\begin{array}{c}\text { First } \\
\text { season }\end{array}$ & $\begin{array}{l}\text { Second } \\
\text { season }\end{array}$ \\
\hline Control & 0.00 & 74.15 & 73.21 & 15.30 & 16.90 & 90.33 & 90.10 & 9.10 & 8.90 \\
\hline \multirow{3}{*}{$\begin{array}{l}\text { Ascorbic } \\
\text { acid }\end{array}$} & 2.5 & 77.25 & 78.00 & 18.10 & 19.10 & 94.90 & 93.55 & 10.36 & 10.40 \\
\hline & 5 & 80.15 & 80.18 & 18.90 & 19.90 & 96.60 & 96.10 & 10.66 & 10.51 \\
\hline & 10 & 84.10 & 84.65 & 26.20 & 26.30 & 105.10 & 106.30 & 12.45 & 12.30 \\
\hline \multirow{3}{*}{$\begin{array}{l}\text { Citric } \\
\text { acid }\end{array}$} & 2.5 & 78.90 & 79.90 & 19.10 & 20.20 & 95.25 & 95.60 & 10.30 & 10.21 \\
\hline & 5 & 79.90 & 81.12 & 22.10 & 23.60 & 97.71 & 96.10 & 10.40 & 10.42 \\
\hline & 10 & 83.18 & 82.96 & 26.10 & 25.50 & 103.66 & 106.10 & 11.60 & 11.85 \\
\hline \multirow{3}{*}{$\begin{array}{l}\text { Oxalic } \\
\text { acid }\end{array}$} & 2.5 & 76.90 & 77.01 & 19.10 & 20.60 & 95.60 & 94.96 & 10.51 & 10.60 \\
\hline & 5 & 79.25 & 79.66 & 23.10 & 23.50 & 98.60 & 98.96 & 10.81 & 11.01 \\
\hline & 10 & 84.00 & 83.93 & 25.30 & 28.90 & 104.10 & 103.66 & 12.30 & 11.90 \\
\hline \multirow{3}{*}{$\begin{array}{l}\text { Salicylic } \\
\text { acid }\end{array}$} & 2.5 & 78.18 & 79.20 & 20.20 & 20.40 & 96.00 & 95.85 & 10.65 & 10.91 \\
\hline & 5 & 80.18 & 81.15 & 24.30 & 23.60 & 99.10 & 99.25 & 10.86 & 10.79 \\
\hline & 10 & 84.09 & 84.33 & 26.15 & 25.95 & 104.20 & 106.20 & 12.40 & 12.21 \\
\hline \multicolumn{2}{|c|}{ L.S.D. at $5 \%$} & 2.59 & 2.73 & 3.01 & 2.89 & 3.50 & 3.89 & 0.67 & 0.69 \\
\hline
\end{tabular}

\section{B- Yield and its components}

Result in Table (3) show that foliar application of antioxidants (Ascorbic acid, Citric acid, Oxalic acid and Salicylic acid) at the rates of 2.5, 5 and $10 \mathrm{mM}$ resulted in a great increase in yield and its components of onion plant. The beneficial effect was more pronounced with the highest rate (10

Fayoum J. Agric. Res. \& Dev., Vol. 21, No. 1, January, 2007 
$\mathrm{mM}$ ) of all antioxidants used. At these treatments diameter of bulbs, fresh and dry weight of bulb and total yield of bulbs/ fed. were significantly increased by $33.33,29.24,28.60$ and $29.89 \%$ in diameter of bulb and 30.02, 29.18, 28.34 and $29.86 \%$ in fresh weight of bulb and $46.99,46.54,45.26$

and $46.80 \%$ in dry weigh of bulb and $35.73,35.27,31.12$ and $35.46 \%$ in total yield of bulb respectively in comparison to the control plants in the first season. Similar trend was observed in the second season.

Table (3): Effect of some antioxidants on yield and its components (diameter of bulb, fresh and dry weight of bulb as well as total yield of bulbs) of onion plants during $2004 / 2005$ and $2005 / 2006$ seasons.

\begin{tabular}{|c|c|c|c|c|c|c|c|c|c|}
\hline \multirow[b]{2}{*}{ Treat. } & \multirow{2}{*}{$\begin{array}{l}\text { Conc } \\
(\mathbf{m M})\end{array}$} & \multicolumn{2}{|c|}{$\begin{array}{l}\text { Diameter of bulb } \\
(\mathrm{cm})\end{array}$} & \multicolumn{2}{|c|}{$\begin{array}{c}\text { Fresh weight of bulb } \\
(\mathrm{g})\end{array}$} & \multicolumn{2}{|c|}{$\begin{array}{l}\text { Dry weight of } \\
\text { bulb (g) }\end{array}$} & \multicolumn{2}{|c|}{$\begin{array}{l}\text { Total yield of bulbs } \\
\text { (ton/fed.) }\end{array}$} \\
\hline & & $\begin{array}{c}\text { First } \\
\text { season }\end{array}$ & $\begin{array}{l}\text { Second } \\
\text { season }\end{array}$ & $\begin{array}{l}\text { First } \\
\text { season }\end{array}$ & $\begin{array}{l}\text { Second } \\
\text { season }\end{array}$ & $\begin{array}{c}\text { First } \\
\text { season }\end{array}$ & $\begin{array}{l}\text { Second } \\
\text { season }\end{array}$ & $\begin{array}{c}\text { First } \\
\text { season }\end{array}$ & $\begin{array}{l}\text { Second } \\
\text { season }\end{array}$ \\
\hline Control & 0.00 & 9.30 & 9.55 & 141.0 & 145.50 & 15.60 & 15.95 & 10.83 & 11.29 \\
\hline \multirow{3}{*}{$\begin{array}{l}\text { Ascorbic } \\
\text { acid }\end{array}$} & 2.5 & 10.60 & 10.67 & 160.11 & 164.25 & 18.41 & 18.60 & 12.32 & 12.40 \\
\hline & 5 & 10.86 & 10.95 & 175.60 & 169.60 & 21.50 & 21.10 & 14.10 & 13.90 \\
\hline & 10 & 12.40 & 12.12 & 183.33 & 183.30 & 22.93 & 23.66 & 14.70 & 14.79 \\
\hline \multirow{3}{*}{$\begin{array}{l}\text { Citric } \\
\text { acid }\end{array}$} & 2.5 & 10.80 & 10.75 & 169.01 & 167.70 & 19.30 & 19.10 & 12.57 & 12.65 \\
\hline & 5 & 10.80 & 11.01 & 178.33 & 180.00 & 21.75 & 21.86 & 14.10 & 14.65 \\
\hline & 10 & 12.02 & 12.10 & 182.15 & 182.33 & 22.86 & 23.10 & 14.65 & 14.73 \\
\hline \multirow{3}{*}{$\begin{array}{l}\text { Oxalic } \\
\text { acid }\end{array}$} & 2.5 & 10.75 & 10.81 & 163.25 & 165.10 & 18.30 & 18.10 & 12.10 & 12.61 \\
\hline & 5 & 11.01 & 11.08 & 173.66 & 175.50 & 21.20 & 21.33 & 13.90 & 13.95 \\
\hline & 10 & 11.96 & 11.90 & 180.96 & 179.99 & 22.66 & 22.18 & 14.20 & 14.73 \\
\hline \multirow{3}{*}{$\begin{array}{l}\text { Salicylic } \\
\text { acid }\end{array}$} & 2.5 & 10.59 & 10.66 & 160.60 & 162.10 & 18.31 & 18.40 & 12.15 & 12.55 \\
\hline & 5 & 10.95 & 10.99 & 175.70 & 176.60 & 21.30 & 21.45 & 13.65 & 13.81 \\
\hline & 10 & 12.08 & 12.11 & 183.10 & 182.80 & 22.90 & 23.40 & 14.67 & 14.76 \\
\hline \multicolumn{2}{|c|}{ L.S.D. at $5 \%$} & 0.67 & 0.64 & 10.80 & 9.73 & 2.01 & 1.85 & 1.60 & 1.03 \\
\hline
\end{tabular}

\section{C- Chemical constituents:-}

\section{1- Leaf pigments:}

Data recorded in Table (4) clearly show in two growing seasons that, foliar application of all antioxidants had a simulating effect on chemical constituents of onion plants. The best results were obtained with the highest rate $(10 \mathrm{mM})$ of ascorbic acid and salicylic acid. In the first season, the increments over the control treatment reached to 43.16 and $43.08 \%$ respectively for chlorophyll a; 39.36 and $39.32 \%$ respectively for chlorophyll b, 52.68 and $52.28 \%$ respectively for caroteniods as well as 21.99 and $19.99 \%$ respectively for anthocyanin compared to the control untreated plants. Similar trend was observed in the second season (Table 4). 
Table (4): Effect of some antioxidants on leaf pigments (chlorophyll a and b as well as total carotenoids and anthocyanin) of onion plants during 2004/2005 and 2005/2006 seasons.

\begin{tabular}{|c|c|c|c|c|c|c|c|c|c|}
\hline \multirow[b]{2}{*}{ Treat. } & \multirow{2}{*}{$\begin{array}{l}\text { Conc } \\
(\mathbf{m M})\end{array}$} & \multicolumn{2}{|c|}{$\begin{array}{l}\text { Chlorophyll a } \\
\text { (ug/g F.W.) }\end{array}$} & \multicolumn{2}{|c|}{$\begin{array}{l}\text { Chlorophyll b } \\
\text { (ug/g F.W.) }\end{array}$} & \multicolumn{2}{|c|}{$\begin{array}{l}\text { Total carotenoids } \\
\text { (ug/g F.W. })\end{array}$} & \multicolumn{2}{|c|}{$\begin{array}{c}\text { Anthocyanin } \\
\text { (mg/100 g D.W.) }\end{array}$} \\
\hline & & $\begin{array}{c}\text { First } \\
\text { season }\end{array}$ & $\begin{array}{l}\text { Second } \\
\text { season }\end{array}$ & $\begin{array}{c}\text { First } \\
\text { season }\end{array}$ & $\begin{array}{l}\text { Second } \\
\text { season }\end{array}$ & $\begin{array}{c}\text { First } \\
\text { season }\end{array}$ & $\begin{array}{l}\text { Second } \\
\text { season }\end{array}$ & $\begin{array}{c}\text { First } \\
\text { season }\end{array}$ & $\begin{array}{l}\text { Second } \\
\text { season }\end{array}$ \\
\hline Control & 0.00 & 266.08 & 283.50 & 225.30 & 226.30 & 29.80 & 27.39 & 50.91 & 51.18 \\
\hline \multirow{3}{*}{$\begin{array}{l}\text { Ascorbic } \\
\text { acid }\end{array}$} & 2.5 & 278.80 & 339.00 & 233.04 & 245.15 & 32.79 & 30.50 & 56.0 & 58.33 \\
\hline & 5 & 326.60 & 353.00 & 246.95 & 251.13 & 42.90 & 33.30 & 59.06 & 59.11 \\
\hline & 10 & 380.93 & 417.25 & 314.00 & 319.15 & 45.50 & 44.70 & 62.11 & 61.63 \\
\hline \multirow[t]{3}{*}{ Citric acid } & 2.5 & 269.33 & 338.23 & 229.33 & 233.25 & 31.1 & 29.99 & 54.10 & 53.25 \\
\hline & 5 & 276.80 & 339.20 & 248.80 & 251.18 & 42.25 & 32.96 & 55.70 & 58.18 \\
\hline & 10 & 378.75 & 409.33 & 278.80 & 290.25 & 43.42 & 36.18 & 60.15 & 59.10 \\
\hline \multirow{3}{*}{$\begin{array}{l}\text { Oxalic } \\
\text { acid }\end{array}$} & 2.5 & 270.00 & 301.60 & 235.66 & 233.33 & 35.32 & 34.43 & 56.15 & 55.21 \\
\hline & 5 & 278.80 & 334.28 & 248.26 & 265.00 & 37.18 & 37.70 & 58.06 & 59.18 \\
\hline & 10 & 380.23 & 394.28 & 313.78 & 306.18 & 43.79 & 39.32 & 59.04 & 59.60 \\
\hline \multirow{3}{*}{$\begin{array}{l}\text { Salicylic } \\
\text { acid }\end{array}$} & 2.5 & 304.28 & 320.18 & 268.26 & 253.15 & 37.42 & 31.99 & 53.60 & 54.26 \\
\hline & 5 & 31.18 & 353.73 & 301.60 & 282.66 & 42.27 & 33.32 & 54.25 & 54.66 \\
\hline & 10 & 380.73 & 413.23 & 313.88 & 309.60 & 45.38 & 43.55 & 61.09 & 60.17 \\
\hline \multicolumn{2}{|c|}{ L.S.D. at $5 \%$} & 38.55 & 34.66 & 28.42 & 30.28 & 10.27 & 3.67 & 3.55 & 3.72 \\
\hline
\end{tabular}

\section{2- Carbohydrates and Sugars:-}

Data recorded in Tables (5 and 6) clearly show that antioxidant application had positive effect on the chemical constituents of onion leaves and bulbs. It is clear that, carbohydrates represented as total carbohydrates, total sugars and reducing sugars increased with increasing the rate of all antioxidants used. The best results were obtained with the highest rate $(10 \mathrm{mM})$ of Ascorbic acid followed by the same rate of Salicylic acid. In the first season, the increments were recorded 16.99 and $16.94 \%$ for total carbohydrates; 27.47 and $25.42 \%$ for total sugars; 24.94 and $24.92 \%$ for reducing sugars in leaves as well as 20.61 and $19.84 \%$ for total soluble solids and 17.92 and $17.45 \%$ for total soluble carbohydrates in bulbs over the control, respectively. Similar trend was observed in the second season. The obtained results in this study showed clearly that chemical constituents of leaves and bulbs of onion plants were greatly improved by antioxidants application especially Ascorbic acid and Salicylic acid.

\section{3-Crude protein \%}

Data recorded in Tables (5 and 6) show that foliar application of all used antioxidants had a stimulating effect on crude protein of onion leaves and bulbs. The concentration of crude protein were gradually increased with increasing the rate of antioxidants concentration. The best results were obtained with the highest rate at $10 \mathrm{mM}$ of ascorbic acid followed by salicylic acid. In the first season, the increases recorded were 62.99 and $62.13 \%$ for crude protein, in leaves and 22.63 and $22.10 \%$ respectively for crude protein in bulbs over the control. Similar trend was observed in the second season. 
EFFECT OF SOME ANTIOXIDANTS ON GROWTH, YIELD.

168

Table (5): Effect of some antioxidants on total soluble carbohydrates, total sugars, reducing sugars and crude protein of onion leaves during $\mathbf{2 0 0 4 / 2 0 0 5}$ and $2005 / 2006$ seasons.

\begin{tabular}{|c|c|c|c|c|c|c|c|c|c|}
\hline \multirow[t]{2}{*}{ Treat. } & \multirow[t]{2}{*}{$\begin{array}{r}\text { Conc } \\
(\mathrm{mM})\end{array}$} & \multicolumn{2}{|c|}{$\begin{array}{l}\text { Total soluble } \\
\text { carbohydrates } \\
\text { (mg/g D.W. ) }\end{array}$} & \multicolumn{2}{|c|}{$\begin{array}{l}\text { Total sugars } \\
\text { (mg/g D.W.) }\end{array}$} & \multicolumn{2}{|c|}{$\begin{array}{l}\text { Reducing sugars } \\
\quad(\mathrm{mg} / \mathrm{g} \text { D.W.) }\end{array}$} & \multicolumn{2}{|c|}{$\begin{array}{l}\text { Crude protein } \\
\text { (g/100 g D.W.) }\end{array}$} \\
\hline & & $\begin{array}{c}\text { First } \\
\text { season }\end{array}$ & $\begin{array}{l}\text { Second } \\
\text { season }\end{array}$ & $\begin{array}{c}\text { First } \\
\text { season }\end{array}$ & $\begin{array}{l}\text { Second } \\
\text { season }\end{array}$ & $\begin{array}{c}\text { First } \\
\text { season }\end{array}$ & $\begin{array}{l}\text { Second } \\
\text { season }\end{array}$ & $\begin{array}{c}\text { First } \\
\text { season }\end{array}$ & $\begin{array}{l}\text { Second } \\
\text { season }\end{array}$ \\
\hline Control & 0.00 & 111.21 & 116.60 & 79.99 & 75.12 & 47.39 & 45.12 & 13.97 & 14.08 \\
\hline $\begin{array}{l}\text { Ascorbic } \\
\text { acid }\end{array}$ & $\begin{array}{l}2.5 \\
5 \\
10 \\
\end{array}$ & $\begin{array}{l}121.55 \\
126.60 \\
130.11 \\
\end{array}$ & $\begin{array}{l}122.22 \\
125.14 \\
132.22 \\
\end{array}$ & $\begin{array}{c}86.73 \\
87.49 \\
101.97 \\
\end{array}$ & $\begin{array}{c}84.18 \\
89.99 \\
101.11 \\
\end{array}$ & $\begin{array}{l}53.20 \\
59.15 \\
59.21 \\
\end{array}$ & $\begin{array}{l}56.16 \\
58.28 \\
60.99 \\
\end{array}$ & $\begin{array}{l}20.35 \\
21.39 \\
22.77 \\
\end{array}$ & $\begin{array}{l}19.09 \\
20.18 \\
22.99 \\
\end{array}$ \\
\hline $\begin{array}{l}\text { Citric } \\
\text { acid }\end{array}$ & $\begin{array}{l}2.5 \\
5 \\
10 \\
\end{array}$ & $\begin{array}{l}118.33 \\
123.36 \\
129.18 \\
\end{array}$ & $\begin{array}{l}121.13 \\
124.20 \\
127.16 \\
\end{array}$ & $\begin{array}{c}81.25 \\
83.33 \\
100.24 \\
\end{array}$ & $\begin{array}{l}81.25 \\
84.16 \\
99.01\end{array}$ & $\begin{array}{l}50.10 \\
52.15 \\
59.18 \\
\end{array}$ & $\begin{array}{l}48.13 \\
50.51 \\
60.16 \\
\end{array}$ & $\begin{array}{l}14.26 \\
16.27 \\
22.08 \\
\end{array}$ & $\begin{array}{l}14.66 \\
17.13 \\
22.08 \\
\end{array}$ \\
\hline $\begin{array}{l}\text { Oxalic } \\
\text { Acid }\end{array}$ & $\begin{array}{l}2.5 \\
5 \\
10 \\
\end{array}$ & $\begin{array}{l}117.27 \\
123.18 \\
129.99 \\
\end{array}$ & $\begin{array}{l}120.16 \\
122.20 \\
126.98 \\
\end{array}$ & $\begin{array}{l}83.67 \\
95.83 \\
97.91 \\
\end{array}$ & $\begin{array}{c}86.28 \\
93.28 \\
100.61 \\
\end{array}$ & $\begin{array}{l}56.44 \\
56.56 \\
59.12 \\
\end{array}$ & $\begin{array}{l}54.14 \\
56.56 \\
60.61 \\
\end{array}$ & $\begin{array}{l}19.20 \\
20.36 \\
22.42 \\
\end{array}$ & $\begin{array}{l}17.07 \\
20.64 \\
22.54\end{array}$ \\
\hline $\begin{array}{l}\text { Salicylic } \\
\text { Acid }\end{array}$ & $\begin{array}{l}2.5 \\
5 \\
10\end{array}$ & $\begin{array}{l}126.18 \\
128.20 \\
130.05\end{array}$ & $\begin{array}{l}126.98 \\
126.15 \\
131.31 \\
\end{array}$ & $\begin{array}{c}81.16 \\
85.41 \\
100.33 \\
\end{array}$ & $\begin{array}{c}83.33 \\
89.26 \\
100.99 \\
\end{array}$ & $\begin{array}{l}51.18 \\
51.21 \\
59.20\end{array}$ & $\begin{array}{l}52.20 \\
54.12 \\
60.70\end{array}$ & $\begin{array}{l}14.26 \\
16.27 \\
22.65\end{array}$ & $\begin{array}{l}15.18 \\
16.96 \\
22.59\end{array}$ \\
\hline L.S.D. at & & 5.59 & 3.74 & 7.73 & 7.62 & 3.64 & 4.16 & 2.74 & 2.58 \\
\hline
\end{tabular}

Table (6): Effect of some antioxidants on total soluble solids (T.S.S.)\%, total soluble carbohydrates \% and crude protein \% of onion bulbs during 2004/2005 and 2005/2006 seasons.

\begin{tabular}{|c|c|c|c|c|c|c|c|}
\hline \multirow{2}{*}{ Treat. } & \multirow[t]{2}{*}{$\begin{array}{r}\text { Conc } \\
(\mathbf{m M})\end{array}$} & \multicolumn{2}{|c|}{$\begin{array}{l}\text { Total soluble solids } \\
\text { (T.S.S.) } \%\end{array}$} & \multicolumn{2}{|c|}{$\begin{array}{c}\text { Total soluble } \\
\text { carbohydrates \% } \\
\text { (D.W. of bulb) }\end{array}$} & \multicolumn{2}{|c|}{$\begin{array}{l}\text { Crude protein \% } \\
\text { (D.W. of bulb) }\end{array}$} \\
\hline & & $\begin{array}{c}\text { First } \\
\text { season }\end{array}$ & $\begin{array}{l}\text { Second } \\
\text { season }\end{array}$ & $\begin{array}{c}\text { First } \\
\text { season }\end{array}$ & $\begin{array}{l}\text { Second } \\
\text { season }\end{array}$ & $\begin{array}{l}\text { First } \\
\text { season }\end{array}$ & $\begin{array}{l}\text { Second } \\
\text { season }\end{array}$ \\
\hline Control & 0.00 & 13.10 & 13.20 & 10.60 & 10.40 & 9.50 & 8.45 \\
\hline $\begin{array}{l}\text { Ascorbic } \\
\text { acid }\end{array}$ & $\begin{array}{l}2.5 \\
5 \\
10\end{array}$ & $\begin{array}{l}13.60 \\
14.60 \\
15.80\end{array}$ & $\begin{array}{l}13.80 \\
14.70 \\
15.41\end{array}$ & $\begin{array}{l}11.90 \\
11.95 \\
12.50\end{array}$ & $\begin{array}{l}12.10 \\
12.15 \\
12.70\end{array}$ & $\begin{array}{l}10.20 \\
11.05 \\
11.65\end{array}$ & $\begin{array}{l}10.30 \\
11.16 \\
11.66\end{array}$ \\
\hline $\begin{array}{l}\text { Citric } \\
\text { acid }\end{array}$ & $\begin{array}{l}2.5 \\
5 \\
10 \\
\end{array}$ & $\begin{array}{l}13.80 \\
14.50 \\
15.10 \\
\end{array}$ & $\begin{array}{l}13.80 \\
14.50 \\
15.60\end{array}$ & $\begin{array}{l}11.85 \\
12.01 \\
12.41\end{array}$ & $\begin{array}{l}11.73 \\
12.31 \\
12.56\end{array}$ & $\begin{array}{l}10.40 \\
11.15 \\
11.35 \\
\end{array}$ & $\begin{array}{l}10.35 \\
11.25 \\
11.41 \\
\end{array}$ \\
\hline $\begin{array}{l}\text { Oxalic } \\
\text { acid }\end{array}$ & $\begin{array}{l}2.5 \\
5 \\
10 \\
\end{array}$ & $\begin{array}{l}13.70 \\
14.90 \\
15.60 \\
\end{array}$ & $\begin{array}{l}13.80 \\
14.70 \\
15.50 \\
\end{array}$ & $\begin{array}{l}11.95 \\
11.99 \\
12.30 \\
\end{array}$ & $\begin{array}{l}12.01 \\
12.23 \\
12.51\end{array}$ & $\begin{array}{l}10.35 \\
11.25 \\
11.55 \\
\end{array}$ & $\begin{array}{l}10.46 \\
11.31 \\
11.59 \\
\end{array}$ \\
\hline $\begin{array}{l}\text { Salicylic } \\
\text { acid }\end{array}$ & $\begin{array}{l}2.5 \\
5 \\
10 \\
\end{array}$ & $\begin{array}{l}13.80 \\
14.00 \\
15.70 \\
\end{array}$ & $\begin{array}{l}13.60 \\
14.20 \\
15.65 \\
\end{array}$ & $\begin{array}{l}11.75 \\
11.95 \\
12.45 \\
\end{array}$ & $\begin{array}{l}11.91 \\
12.03 \\
12.66 \\
\end{array}$ & $\begin{array}{l}10.25 \\
11.25 \\
11.60 \\
\end{array}$ & $\begin{array}{l}10.38 \\
11.29 \\
11.62 \\
\end{array}$ \\
\hline L.S.D. at & & 0.69 & 0.74 & 0.40 & 0.49 & 0.56 & 0.57 \\
\hline
\end{tabular}

\section{4-Total free amino acids and total indoles concentration:-}

Data in both two seasons presented in Table (7) indicate that, leaves of onion plants contained a high concentration of total free amino acids and total indoles under antioxidants applications comparing to control plants. Moreover,

Fayoum J. Agric. Res. \& Dev., Vol. 21, No. 1, January, 2007 
the total free amino acids and total indoles were significantly increased with increasing antioxidants rates. The highest rate gave the best results especially ascorbic acid and salicylic acid at the rate $(10 \mathrm{mM})$; the maximum increase was 51.27 and $47.87 \%$ in the first season and 42.76 and $42.46 \%$ in the second seasons for total free amino acids, respectively and 58.42 and $52.80 \%$ in the first season and 53.33 and $51.11 \%$ in the second seasons for total indoles respectively over the control plants.

\section{5- Total soluble phenols:-}

Data recorded in Table (7) clearly show total soluble phenols of leaves were greatly affected by the different kinds and levels of antioxidants application. Moreover, the total soluble phenols were significantly increased with increasing antioxidants rates. The maximum increase in total phenols concentration reached 51.27 and $45.20 \%$ for ascorbic acid and salicylic acid treatments at the rate $(10 \mathrm{mM})$ in the first seasons and 45.43 and 44.16 in the second seasons respectively over the control.

Table (7): Effect of some antioxidants on total free amino acids, total indoles and total soluble phenols of onion leaves during 2004/2005 and 2005/2006 seasons.

\begin{tabular}{|c|c|c|c|c|c|c|c|}
\hline \multirow[t]{2}{*}{ Treat. } & \multirow[t]{2}{*}{$\begin{array}{l}\text { Conc } \\
(\mathrm{mM})\end{array}$} & \multicolumn{2}{|c|}{$\begin{array}{c}\text { Total free amino acids } \\
\text { ( } \mathrm{mg} / \mathrm{g} \text { D.W. })\end{array}$} & \multicolumn{2}{|c|}{$\begin{array}{l}\text { Total indoles } \\
\text { (mg/g F.W.) }\end{array}$} & \multicolumn{2}{|c|}{$\begin{array}{c}\text { Total soluble phenols } \\
\text { (mg/g D.W.) }\end{array}$} \\
\hline & & $\begin{array}{l}\text { First } \\
\text { season }\end{array}$ & $\begin{array}{l}\text { Second } \\
\text { Season }\end{array}$ & $\begin{array}{l}\text { First } \\
\text { season }\end{array}$ & $\begin{array}{l}\text { Second } \\
\text { season }\end{array}$ & $\begin{array}{c}\text { First } \\
\text { season }\end{array}$ & $\begin{array}{l}\text { Second } \\
\text { season }\end{array}$ \\
\hline Control & 0.00 & 14.46 & 16.39 & 0.89 & 0.90 & 5.11 & 4.71 \\
\hline \multirow{3}{*}{$\begin{array}{l}\text { Ascorbic } \\
\text { acid }\end{array}$} & 2.5 & 18.42 & 18.21 & 1.10 & 1.22 & 6.44 & 4.92 \\
\hline & 5 & 19.40 & 19.21 & 1.19 & 1.24 & 6.44 & 5.68 \\
\hline & 10 & 24.90 & 23.40 & 1.41 & 1.38 & 7.73 & 6.85 \\
\hline \multirow{3}{*}{$\begin{array}{l}\text { Citric } \\
\text { acid }\end{array}$} & 2.5 & 19.40 & 18.10 & 0.91 & 0.93 & 5.11 & 5.01 \\
\hline & 5 & 19.60 & 19.10 & 1.19 & 1.22 & 6.11 & 5.77 \\
\hline & 10 & 23.43 & 23.20 & 1.20 & 1.26 & 7.33 & 6.68 \\
\hline \multirow{3}{*}{$\begin{array}{l}\text { Oxalic } \\
\text { acid }\end{array}$} & 2.5 & 20.46 & 21.80 & 0.91 & 0.95 & 5.33 & 4.44 \\
\hline & 5 & 21.34 & 21.9 & 1.10 & 1.08 & 6.89 & 5.70 \\
\hline & 10 & 24.33 & 22.90 & 1.31 & 1.26 & 7.33 & 5.83 \\
\hline \multirow{3}{*}{$\begin{array}{l}\text { Salicylic } \\
\text { acid }\end{array}$} & 2.5 & 19.94 & 20.90 & 0.85 & 0.94 & 5.78 & 4.78 \\
\hline & 5 & 21.37 & 21.13 & 0.89 & 0.94 & 5.79 & 5.79 \\
\hline & 10 & 24.34 & 23.35 & 1.36 & 1.36 & 7.42 & 6.79 \\
\hline \multicolumn{2}{|c|}{ L.S.D. at $5 \%$} & 4.81 & 4.78 & 0.164 & 0.143 & 0.73 & 0.72 \\
\hline
\end{tabular}

\section{6- Nitrogen, phosphorus and potassium concentrations:-}

Data in both two seasons presented in Table (8) indicate that, leaves of onion plants contained a high concentration of nitrogen, phosphorus and potassium under antioxidants applications condition comparing to control untreated plants. Moreover, the concentration of these elements were significantly increased with increasing antioxidants rates. The maximum increase which obtained from ascorbic acid and salicylic acid treatments at the rate of $(10 \mathrm{mM})$ were 62.96 and $62.14 \%$ for nitrogen concentration; 51.85 and $48.15 \%$ for phosphorus concentration and 51.85 and $46.03 \%$ for potassium concentration in the first season respectively over the control plants. Similar trend was observed in the second season, Table (8).

Fayoum J. Agric. Res. \& Dev., Vol. 21, No. 1, January, 2007 
EFFECT OF SOME ANTIOXIDANTS ON GROWTH, YIELD.

Table (8): Effect of some antioxidants on total nitrogen, phosphorus and potassium of onion leaves during 2004/2005 and 2005/2006 seasons.

\begin{tabular}{|c|c|c|c|c|c|c|c|}
\hline \multirow[t]{2}{*}{ Treat. } & \multirow{2}{*}{$\begin{array}{l}\text { Conc } \\
(\mathbf{m M})\end{array}$} & \multicolumn{2}{|c|}{ Total nitrogen $(\%)$} & \multicolumn{2}{|c|}{ Total phosphorus (\%) } & \multicolumn{2}{|c|}{ Total potassium $(\%)$} \\
\hline & & $\begin{array}{c}\text { First } \\
\text { season }\end{array}$ & $\begin{array}{l}\text { Second } \\
\text { season }\end{array}$ & $\begin{array}{c}\text { First } \\
\text { season }\end{array}$ & $\begin{array}{l}\text { Second } \\
\text { season }\end{array}$ & $\begin{array}{c}\text { First } \\
\text { season }\end{array}$ & $\begin{array}{l}\text { Second } \\
\text { season }\end{array}$ \\
\hline Control & 0.00 & 2.43 & 2.45 & 0.27 & 0.25 & 1.89 & 1.91 \\
\hline \multirow{3}{*}{$\begin{array}{l}\text { Ascorbic } \\
\text { acid }\end{array}$} & 2.5 & 3.54 & 3.32 & 0.29 & 0.29 & 2.51 & 2.46 \\
\hline & 5 & 3.72 & 3.51 & 0.38 & 0.36 & 2.56 & 2.61 \\
\hline & 10 & 3.96 & 3.99 & 0.41 & 0.39 & 2.87 & 2.91 \\
\hline \multirow{3}{*}{$\begin{array}{l}\text { Citric } \\
\text { acid }\end{array}$} & 2.5 & 2.48 & 2.55 & 0.30 & 0.31 & 2.43 & 2.45 \\
\hline & 5 & 2.83 & 2.98 & 0.35 & 0.36 & 2.55 & 2.57 \\
\hline & 10 & 3.84 & 3.84 & 0.39 & 0.37 & 2.71 & 2.75 \\
\hline \multirow{3}{*}{$\begin{array}{l}\text { Oxalic } \\
\text { acid }\end{array}$} & 2.5 & 3.34 & 2.97 & 0.29 & 0.30 & 2.36 & 2.41 \\
\hline & 5 & 3.54 & 3.59 & 0.31 & 0.33 & 2.58 & 2.60 \\
\hline & 10 & 3.90 & 3.92 & 0.37 & 0.37 & 2.66 & 2.70 \\
\hline \multirow{3}{*}{$\begin{array}{l}\text { Salicylic } \\
\text { acid }\end{array}$} & 2.5 & 2.48 & 2.64 & 0.32 & 0.31 & 2.31 & 2.29 \\
\hline & 5 & 2.83 & 2.95 & 0.36 & 0.36 & 2.65 & 2.68 \\
\hline & 10 & 3.94 & 3.93 & 0.40 & 0.38 & 2.76 & 2.79 \\
\hline \multicolumn{2}{|c|}{ L.S.D. at $5 \%$} & 0.53 & 0.41 & 0.038 & 0.035 & 0.20 & 0.21 \\
\hline
\end{tabular}

\section{DISCUSSION}

It is clear from the results of the present study that, foliar spray of antioxidants; i.e. (ascorbic acid, citric acid, oxalic acid and salicylic acid) recorded the highest value on all of the studied growth characters (plant height etc........), yield and its components and chemical constituents of leaves and bulbs (chlorophyll a, b, caroteniods, sugars, anthocyanin, total free amino acid, crude protein, total soluble phenols, total indoles, and macro elements $(\mathrm{N}, \mathrm{P}$ and $\mathrm{K}$ concentration). The beneficial effect of antioxidants on growth characters may be attributed to their positive effect on enhancing cell division and counteracting drought, salinity and diseases stresses and protecting plant cells against free radicals that responsible for plant senescence as well as to their auxinic action (Prusky, 1988; Elade, 1992 and Raskin, 1992). In addition, ascorbic acid maybe regulate cell wall expansion, cell division and cell elongation through its action in cell vacuolarization (Gonzalez-Reyes $\boldsymbol{e t}$ al., 1994; Navas and Gomez-Diaz, 1995 and Cordoba-Pedregosa et al., 1996), that might improve the nutritional status and absorbing phenolic compounds which lead to save the growing tissues from toxic effects of the oxidized phenols (Gupta et al., 1980) and/or enhances the biosynthesis of carbohydrates and translocation of sugars (Ahmed, 2001) which could explain the results of this study. In this respect, Shahda, (2000) reported that ascorbic acid, benzoic acid, or salicylic acid exhibited growth promoting effect and subsequently increased the shoot and root growth as well as fresh and dry weight. These findings are in coincidence with those obtained by Ahmed et al. (1998), Tarraf et al. (1999), Mostafa (2004) and Rady (2006). Also the improving effect of antioxidant on onion yield and its components may be mainly attributed to its positive action on enhancing growth parameters (Table 3 ), photosynthetic pigments of plants leaves, cellular solutes, i.e. total sugars, total free amino aced, total indoles, to sustenance of cell turgor leading to maintenance of metabolic activities in plants and plant nutritional status. In

Fayoum J. Agric. Res. \& Dev., Vol. 21, No. 1, January, 2007 
this respect, Datta and Nanda (1985) reported that the effect of salicylic acid may be brought about through an increase in mobilization of reserve food materials. Also, Al-Qubaie (2002) stated that ascorbic acid as an antioxidant compound has an auxinic action and also synergistic effect on the biosynthesis of carbohydrates and controlling the incidence of most fungi on plants makes them in vigorous states and reflects on seed yield and production. Besides, the induced effect of ascorbic acid as one of vitamins on oil content may be due to that vitamins are recognized to be coenzymes involved in specific biochemical reactions in plants such as oxidative and nonoxidative decarboxylations (Robinson, 1973). Furthermore, Shalaby (2006) reported an increase in yield bulbs of onion plant as a result of the foliar application with some antioxidants. Their results regarding the beneficial effect of ascorbic acid on yield are confirmed with those reported by Ahmed et al. (2002), Mostafa (2004) and Rady (2006). The results also showed that high concentrations of chemical constituents estimated in the leaves and bulbs of the above mentioned treated plants. This finding could be an indication to the high rate of synthesis of these products as indication to the high level of absorption of the essential nutrients for the various growth processes such as chlorophyll formation, photosynthetic activity as well as the utilization of carbohydrates. In this connection, Foyer et al.(1990) stated that, the antioxidant prevent enzymes inactivation, the generation of more dangerous radicals and allow flexibility in the production of photosynthetic assimilatory power. The role of antioxidant, which directly involved in the regulation and protection of photosynthetic processes, could be led to the enhancing effect of antioxidants on photosynthetic pigment. The results obtained in this study are in agreement with those obtained by Farago and Brunhold (1994). In additions Elade (1992) and Abdou et al., (2001) proved that most antioxidants were responsible for accelerating the biosynthesis of various pigments and consequently the photosynthetic rates.. The positive effects of ascorbic acid on photosynthetic pigments obtained by Tarraf et al. (1999), Ali (2002) and AlQubaie (2002) were in agreement with findings of the present study. Concerning the effect of the tested antioxidants on total carbohydrates, total soluble sugars, total soluble solid and anthocyanin data showed that the used antioxidants significantly increased these components. In this respect, Farag (1996) pointed out that most antioxidants were responsible for accelerating the biosynthesis of various pigments leading to the increase in biosynthesis of sugars. Moreover, Al-Qubaie (2002) reported that ascorbic acid as an antioxidant compound has an auxinic action and also synergistic effect on biosynthesis of carbohydrates. Also Omer (1999) reported that, antioxidant increased photosynthesis, leading to an enhancement of the syntheses and accumulation of sugars so, increased T.S.S. Moreover, Tsuda et al. (1994) show that anthocyanins may play an important role in the prevention of lipid peroxidation of cell membranes induced by active oxygen radicals. The increase in the total free amino acid and protein concentration in the treated onion plants with the antioxidants in the present study agreed with those obtained by Abd El-Magid et al., (2004) and Zaghlool et al., (2006). In this respect, Doares $\boldsymbol{e l}$ al., (1995) reported that salicylic acid inhibits synthesis of proteinase inhibitors in tomato leaves induced by systemin and jasmonic acid. Also Reda et al., (1977) reported the involvement of ascorbic acid in many physiological and biochemical processes such as synthesis of enzyme, nucleic acid and protein as well as it also acted as co-enzyme. Moreover, many

Fayoum J. Agric. Res. \& Dev., Vol. 21, No. 1, January, 2007 
investigation mentioned that the hormonal balance between the promoters and inhibitors which regulate the different physiological processes in the plants appear to be highly affected by the antioxidant kind and rates. In this connection, Wafaa et al. (1996) concluded that applied salicylic acid induced great changes in endogenous phytohormones of tomato plants and other plants. Also, Shehata et al. (2000) reported that salicylic acid application increased auxin, cytokinin and gibberellin in cotton leaves. In this respect, cytokinin retarded leaves senescence and increased chlorophyll pigments (Zayed, 1986). Moreover, the increase in phenolic compounds of plants (Table, 4) resulted from an increase in antioxidants thus, a linear relationship between total antioxidants and total phenolic content in plants was found (Campeau and Proctor, 2003). Phenolic antioxidants play an important roles as free radical terminators and sometimes, as metal chelators (Shahidi and Wanasundara, 1992). The results obtained in this study are in harmony with those reported by Ali (2001) and (2002), Al-Qubaie (2002) and Ahmed et al. (2003). Concerning the effect of antioxidants on leaf macronutrients concentration, the data show that all of the tested antioxidants increased significantly leaf mineral concentration. In this respect, Ahmed and Abd ElHameed (2004) reported that the effect of antioxidants on producing healthy plants leads to enhancement of the uptake of mineral elements. Moreover, Gonzalez-Reyes et $\boldsymbol{a l}$. (1994) concluded that ascorbate free radical caused hyperpolarization of plasma membranes, and this energization could then facilitate transport processes across such membranes. Most of the previous results are consistent with those of Ali (2001), Ahmed (2001), Ahmed et al. (2002) and Mostafa (2004).

\section{REFERENCES}

Abd El-Magid, M.S.; Abd El-Monem, S.M. and Ibrahim, N.A. (2004): Effect of some antioxidants on white rot in onion and garlic and their yield. Egypt. J. Agric. Res., 82 (3): 1059-1073.

Abdou, E.; Abd-Alla, H.M. and Galal, A.A. (2001): Survey of seasame root rot /wilt diseases in Minia and their possible control by ascorbic acid and salicylic acid. Assiut J. of Agric. Sci., 32(2): 135-152.

Ahmed, A.M. (2001). Studies for controlling malformation and improving yield and fruit quality of hindy bisinnara mangoes by using active dry yeast, ascorbic acid and sulphur. Minia J. of Agric. Res. \& Develop., 21 (2): 219233.

Ahmed, F.F.; Abdalla, A.S. and Sabour, A.M.T. (2003). Growth and fruiting of williams banana as affected by some antioxidant and biofertilizer treatments. Minia J. of Agric. Res. \& Develop., 23 (1): 51-68.

Ahmed, F.F. and Abd El-Hameed, H.M. (2004). Influence of some antioxidants on growth, vine nutritional status, yield and quality of berries in banaty grapevines. Assiut J. of Agric. Sci., 35 (4): 131-140.

Ahmed, F.F.; Darwish, O.H.; Gobara, A.A. and Ali, A.H. (1998). Growth, nutritional status and productivity of Flame seedless grapevine as influenced by the application of citrine and ascobine. Amer. Soc. of Agron. Crops Sci., Soc. of America, Soil Sci., Soc. of America Baltimcre, Maryland (18-22 Oct.) p. 252.

Fayoum J. Agric. Res. \& Dev., Vol. 21, No. 1, January, 2007 
Ahmed, F.F.; Darwish, O.H.; Gobara, A.A. and Ali, A.H. (2002). Physiological studies on the effect of ascorbic and citric acids in combined with some micronutrients on Flame seedless grapevine. Minia J. of Agric. Res. \& Develop., 22 (1): 105-114.

Ali, Z.A. (2001): Ascorbic acid induced anatomical changes in leaves and stems of tomato plants. Bull. NRC Egypt, 26(3): 371-382.

Ali, Z. A. (2002). Effect of foliar application with ascorbic acid on vegetative growth and some biochemical constituents of tomato plants. J. Agric. Sci., Mansoura Univ., 27 (10): 6765-6775.

Al-Qubaie, A. I. (2002). Response of Ficus nitida L. seedlings to the application of some antioxidants under soil salinity conditions. Minia J. of Agric. Res. \& Develop., 22 (3): 235-254.

A.O.A.C. (1995). Official Methods of Analysis of the Association of Official Agricultural Chemists. Sixteenth ed., Washington D.C., USA.

Buchala, A.J. and Schmid, A. (1997). Vitamin D and its analoguous as new class of plant growth substances affecting Rhizogenesis, Nature pp. 280-330.

Campeau, C. and Proctor, J.T.A. (2003). Rust-spotted North American ginseng roots: Phenolic, antioxidant, ginsenoside and mineral nutrient content. Hort. Sci., 38 (2): 179-182.

Cordoba-Pedregosa, M.C.; Gonzalez-Reyes, J.A.; Sandillas, M.S.; Navas, P. and Cordoba, F. (1996). Role of apoplastic and cell-wall peroxidases on the stimulation of root elongation by ascorbate. Plant Physiol., 112: 1119-1125.

Datta, K.S. and Nanda, K.K.(1985). Effect of some phenolic compounds and gibberellic acid on growth and development of Cheena millet (Ponicum millacceum L.). Indian J. of Plant Physiol., 28: 298-302.

Doares, S.H.; Narvaez-Vasquez, J.; Conconi, A. and Ryan, C.A. (1995): Salicylic acid inhibits synthesis of proteins inhibitors in tomato leaves in doused by systemin and jasmonic acid . Plant Physiol., 108 :1741-1746.

Elade, Y. (1992). The use of antioxidants to control gray mould (Botrytis cineria) and white mould (Sclerotinia sclerotiorum L.) in various crops. Plant Pathol., 141: 417-426.

El-Sayed, A.M. and Attia, A.A.M. (1999). A comparative study on some onion (Allium cepa L.) cultivars. Egypt. J. Hort., 26(1): 67-75.

Farago, S. and Brunhold, C. (1994). Regulation of thiol contents in maize roots by intermediates and effectors of glutathione synthesis. J. Plant Physiol., 144: 433-437.

Foyer, C.H.; Furbank, R.T.; Harbinson, J. and Horton, P. (1990). The mechanism contributing to photosynthetic control of electron transport by carbon assimilation in leaves. Photosynth. Res., 25: 83-100.

Gad El-Hak, S.H.; El-Shobaky, S.A.; Galal, A.A. and Zaki, H.E. (2003): Studies on using microdoses of boron, manganese, ascorbic acid and salicylic acid on in vitro potato culture. Minia J. of Agric. Res. \& Develop. 23, 1: 147166.

Gomez, K.A. and Gomez, A.A. (1983): Statistical Analysis Procedure of Agricultural Research. John Wiley \& Sons, New York, pp. 25-30.

Gonzalez-Reyes, J.A.; Alcain, F.J.; Caler, J.A.; Serrano, A.; Cordoba, F. and Navas, P. (1994). Relationship between apoplastic ascorbate regeneration and stimulation of root growth in Allium cepa L. Plant Sci., 100: 23-29.

Gupta, P.K.; Nadgir, A.L.; Macarentias, A.F. and Jagannathan, V. (1980). Tissue culture of forest trees: Clonal multiplication Tecoma grandis L. (treak) by tissue culture. Plant Sci. Letters, 17: 259-268.

Fayoum J. Agric. Res. \& Dev., Vol. 21, No. 1, January, 2007 
EFFECT OF SOME ANTIOXIDANTS ON GROWTH, YIELD

Herbert, D.; Phipps, P.J. and Strange, R.F. (1971). Determination of total carbohydrates. Methods in Microbian, 5 (B): 209-244.

Hoagland, R.E. (1980). Effect of glycophosphate on metabolism of phenolic compounds. VI. Effect of glyphosime and glyphosate metabolites on phenylalanine ammonia lyase activity, growth, protein ,chlorophyll and anthocyanin levels in soybean seedlings. Weed Sci., 28: 393-409.

Ismail, H.A. and Fawzy, A.M. (2001): Effect of salicylic acid and propylgallate on antinutritional factors in pea (Pisum sativum L.) seeds. Minia of Agric., Res. \& Develop., 21 (3): 333-363.

Jayarman, J. (1981): Laboratory Manual in Biochemistry. Wiley Eastern Limited New York, pp. 61-73

Larson, P.; Harbo, A.; Klunsour, S. and Aasheim, T. (1962): On the biogenesis of some indole compounds in Acetobacter xylimum. Physiol. Plant., (15): 552565.

Mostafa, E.A.M. (2004). Effect of spraying with ascorbic acid, vitamin B and active dry yeast on growth, flowering, leaf mineral status, yield and fruit quality of Grand nain banana plants. Annals Agric. Sci., Ain Sha. Univ., Cairo, 49 (2): 643-659.

Navas, P. and Gomez-Diaz, C. (1995). Ascorbate free radical and its role in growth control. Protoplasm, 184: 8-13.

Omer, A. Kh. (1999): Response of Red Roomy Grapevines( Vitis vinifera L.) to some antioxidants and biofertilizer treatments. pp 86-98. M. Sc. Thesis Fac. Agric., Minia Univ., Egypt.

Page, A.I.; Miller, R.H. and Keeny, D.R. (1982). "Methods of Soil Analysis". Part II. Chemical and Microbiological Methods. $2^{\text {nd }}$ Ed. Amer. Soc. Agron., Madison, Wisconsin, USA.

Prusky, D. (1988). The use of antioxidants to delay the onset of anthracnose and stem end decay in avocado fruits after harvest. Plant Disease, 72: 381-384.

Rady, M.M. (2006): Efficiency of growth and productivity of sunflower plants as affected by ascorbic acid under saline reclaimed soil conditions. The Second Conference on Farm Integrated Pest Management, 16-18 Jan., pp. 186-200.

Raskin, I. (1992): Salicylate, a new plant hormone. Plant Physiol., (99): 799-803.

Reda, F.; Fadl, M.; Abdel-All, R. and El-Moursi, A. (1977). Physiological studies on Ammi visnaga $\mathrm{L}$. The effect of thiamine and ascorbic acid on growth and chromone yield. Egypt. J. Pharm. Sci., 18: 19-27.

Robinson, F.A. (1973). Vitamins. "Phytochemistry", Vol. III: 195-198. Lawrence P. Miller (Ed.), Van-Nostrand Reinhold Co., New York.

Shahda, W.T. (2000). The use of antioxidants for control of tomato damping off. Alex. J. of Agric. Research, 45(1):307-316

Shahidi, F. and Wanasundara, P.K. (1992). Phenolic-antioxidants. Crit. Rev. Food Sci. Nutr., 32: 67-103.

Shalaby, O.Y. (2006): Effect of some antioxidants and ridomil on the incidence of onion downy mildew, purple blotch and plant chemical components. Egypt., J. of App. Sci., 21(3): 59-70.

Shehata, S. A. M.; Saeed, M.A. and Abou El-Nour, M. S. (2000). Physiological response of cotton plant to the foliar spray with salicylic acid. Annals Agric.Sci. Ain Shams Univ. Cairo, 45(1): 1-18.

Tarraf, S.A.; Gamal El-Din, K.M. and Balbaa, L.K. (1999). The response of vegetative growth, essential oil of lemongrass (Cymbopogon citrates L.) to foliar application of ascorbic acid, nicotinamide and some micronutrients. Arab Univ. J. Agric. Sci., Ain Shams Univ., Cairo, 7(1): 247-261.

Fayoum J. Agric. Res. \& Dev., Vol. 21, No. 1, January, 2007 
Tsuda, T.; Watanabe, M.; Ohshima, K.; Norinoba, S.; Choi, S.; Kawakishi, S. and Osawa, T. (1994). Antioxioxidative activity of the anthocyanin pigments cyanidin3-O-B-D-glucoside and cyaniding. J. Agric. Food Chem., 42: 24072410.

Wafaa, M. Abdel-Said, N.Y. Abdel- Ghafar, N.Y. and Shehata, S.A.M. (1996). Application of salicylic acid and aspirin for induction of resistance to tomato plants against bacterial wilt and its effect on endogenous hormones. Annals Agric. Sci. Ain Shams Univ., Cairo, 41(2): 1007-1020.

Welburn, A.R. and Lichtenthaler, H. (1984). Formula and program to determine total caroteniods and chlorophyll $\mathrm{a}$ and $\mathrm{b}$ of leaf extracts in different solvents. In Advances in Photosynthesis Research (Sybesma C.Ed.) Vol., II pp. 9- 12.

Wilde, S.A.; Corey, R.B.; Lyer, J.J. and Voigt, G.K. (1985): Soil and Plant Analysis For Tree Culture 3rd Ed. Oxford IBLT Publishing Co., New Delhi: 9-100

Zaghlool, Sanaa, A.M.; Ibrahim, A.M. and Sharaf El-Deen, H.A.M. (2001): The effect of naphthalene acetic acid (NAA), salicylic acid (SA) and their combination on growth, fruit setting, yield and some correlated components in dry bean (Phaseolus vulgaris L.). Annals Agric. Sci. Ain Shams Univ, Cairo 46 (2): 451-463.

Zaghlool, Sanaa, A.M.; Mostafa, M.A. and Shehata, S.A.M. (2006): Physiological studies on the effect of kinetin and salicylic acid on growth and yield of wheat plant. Annals Agric. Sci. Ain Shams Univ., Cairo 51(1): 41-55.

Zayed, A.El. (1986): Comparative studied on the effect of some auxin transport inhibitors on cucumber (Cucumis sativus L.) Angew. Botanik, 60: 23-29.

Zhang, S. and Klessing, D.F. (1997): Salicylic acid activites a 48-KoMAP Kinase in tobacco. Plant Cell , 9: 409-424.

Fayoum J. Agric. Res. \& Dev., Vol. 21, No. 1, January, 2007 


\author{
تأثير بعض مضادات الأكسدة على النمو والمحصول

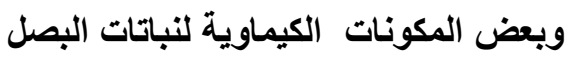

محمد احمد سيف اليزل

قسم النبات الزراعي- كلية الزراعة جمد جامعة الفيوم- مصر

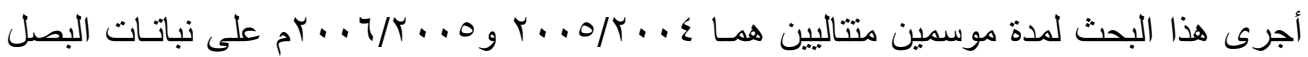

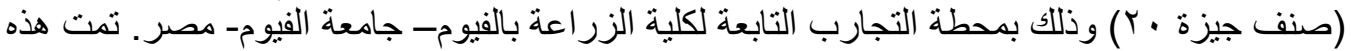

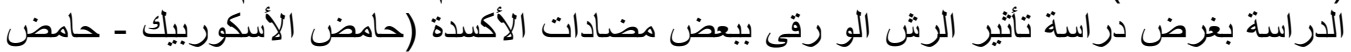

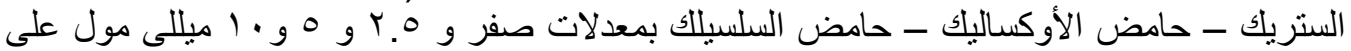

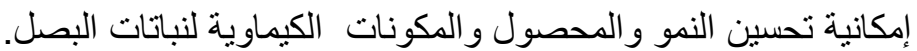

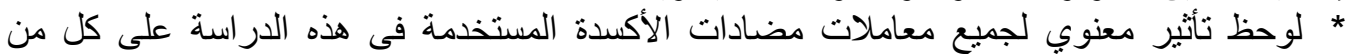

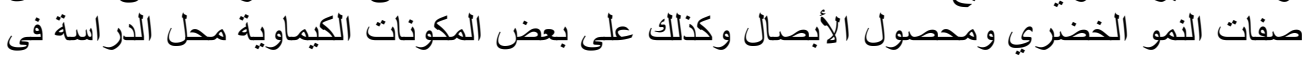

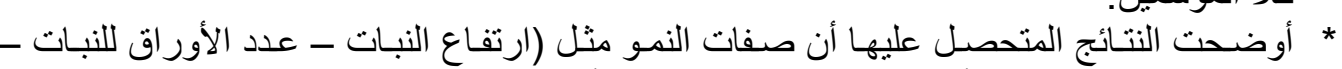

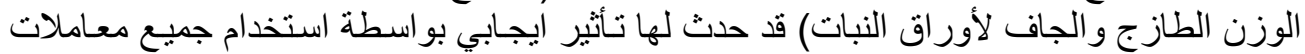

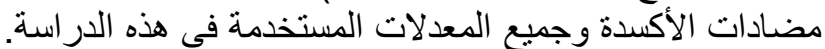

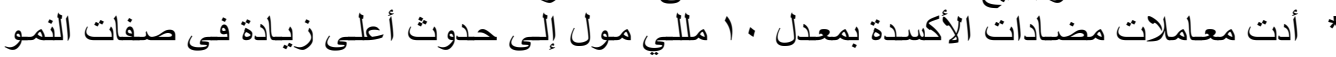

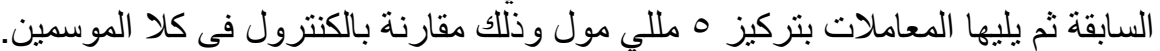

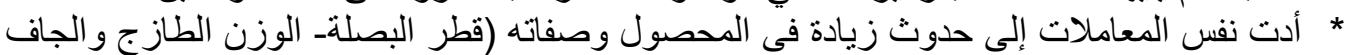

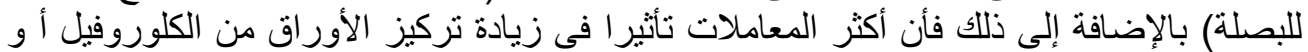

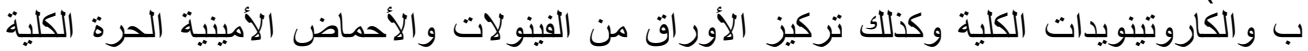

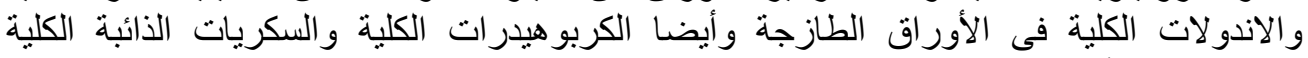

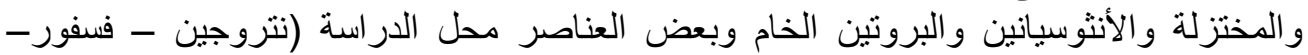

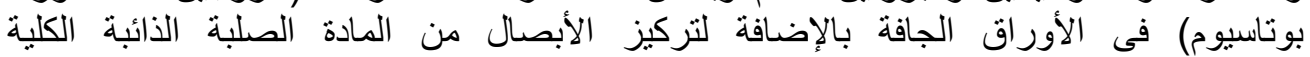

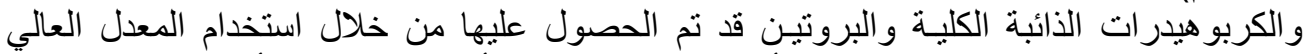

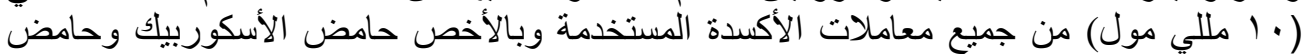

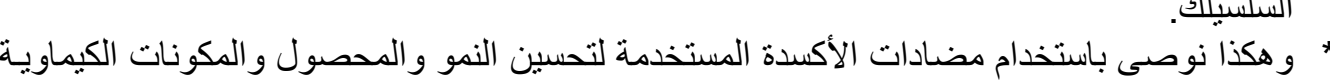

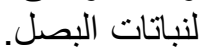

